\title{
Uso del lenguaje nativo en la enseñanza del idioma Inglés en la FAREM Estelí-UNAN Managua
}

\section{Use of native language in the teaching of the English language in FAREM Estelí-UNAN Managua}

\author{
Luis Antonio Arauz Valenzuela ${ }^{1}$
}

\section{RESUMEN}

Durante el siglo XX, discusiones y debates profesionales y metodológicos dentro de la Enseñanza del Lenguaje Ingles (ELT), asumen que el inglés es mejor enseñado y aprendido sin la utilización del idioma del estudiante. Sin embargo, esta asunción se ha ido cuestionando, y el uso de la lengua nativa del estudiante está siendo reevaluada. Existen lagunas en nuestro conocimiento y comprensión sobre la medida en qué, y cómo, el propio lenguaje del estudiante se utiliza dentro de las aulas de clase y las actitudes que presentan los profesores hacia el uso del lenguaje nativo. En el presente escrito, se abordan opiniones de estudios relacionados al tema, antecedentes y las nuevas teorías acerca de ELT. De la misma manera, se analiza el tema del método monolingüe y el método bilingüe y se comparte la experiencia docente, se ejemplifica y se compara la opinión de diversos autores para el entendimiento de tema.

Palabras clave: ELT; lenguaje nativo; monoligue; bilingue.

\begin{abstract}
During the 20th century, discussions and professional and methodological debates within the English Language Teaching (ELT), assume that English is better taught and learned without the use of the student's language. However, this assumption has been questioned, and the use of the student's native language is being reevaluated. There are gaps in our knowledge and understanding of the extent to which, and how, the student's own language is used within classrooms and the attitudes teachers present toward the use of native language. In the present writing, opinions of studies related to the subject, antecedents and the new theories about ELT are addressed. In the same way, the subject of the monolingual method and the bilingual method are analyzed and the teaching experience is shared, the opinion of different authors is exemplified and compared for the understanding of the subject.
\end{abstract}

Keywords: English Language Teaching; native language; monolingual; bilingual.

DOI: https://doi.org/10.5377/multiensayos.v3i6.9682

Recibido: 15 de diciembre de 2017

Aceptado: 05 de enero de 2018

1 Docente UNAN-Mangua/FAREM-Estelí. Correo electrónico: thonyarauz@gmail.com 


\section{INTRODUCCIÓN}

La enseñanza del idioma extranjero (ingles) en la actualidad, es una exigencia que se necesita en cualquier lugar del mundo. Hoy en día la Facultad Regional Multidisciplinaria Estelí UNANMANAGUA, posee maestros especializados en la enseñanza del idioma Inglés. Por tal motivo en la enseñanza de este idioma se deben tomar en cuenta factores que ayuden a los estudiantes a lograr un aprendizaje significativo dentro y fuera de las aulas de clase, estos factores se estarán abordando en este ensayo.

Un factor que se tomara muy en cuenta es la utilización del Lenguaje Nativo dentro de las aulas, en el que se exponen diferentes conceptos y teorías que se han venido creando en este siglo acerca de su utilización o no utilización en la enseñanza-aprendizaje del idioma Ingles.

He observado que en esta Facultad, los profesores que enseñan inglés son hispanohablantes, por lo que comparten la lengua nativa con los alumnos. El inglés se enseña como un idioma extranjero y se pretende la no utilización del español dentro de las aulas de clase.

\section{DESARROLLO}

\section{¿Pero, cómo logramos que los alumnos no utilicen su lenguaje nativo en el aprendizaje del inglés?}

El profesor Guy Cook (2001) expone lo siguiente; “como pretendemos la no utilización del L1 si los estudiantes siguen pensando en su lenguaje nativo y luego exponen oralmente el inglés". Esta fue una de las principales ideas que me llevo a la realización de este ensayo.

\section{El Uso de Lenguaje Nativo (L1) en las clases de Inglés}

El uso de la lengua materna "Lenguaje Nativo (L1)" en el aprendizaje de Inglés es un debate de no acabar dentro de las aulas de clase de Lengua Extranjera. Defensores del método monolingüe sugieren que el lenguaje en estudio debería ser el único medio de comunicación, con la creencia que la prohibición del lenguaje nativo maximizara la eficacia de adquirir dicho idioma. Sin embargo, algunos maestros creen que la utilización de la lengua materna puede ser de mucha ayuda en la adquisición de nuevo vocabulario y en la explicación de ideas complejas y gramáticas necesaria. Estos también disputan que los maestros que dominan el lenguaje nativo de los estudiantes tienen muchas más ventajas sobre los maestros que no lo hacen.

\section{El método monolingüe}

En defensa del método monolingüe, Krashen (1981) argumenta que los estudiantes adquieren un lenguaje extranjero (L2) siguiendo básicamente los mismos pasos que utilizaron en la adquisición 
de la lengua nativa (L1). De acuerdo a él, la utilización de la lengua materna debería ser mínima. De hecho, muchos maestros creen que la lengua materna utilizada en la enseñanza del inglés debería ser minimizada por muchas razones. Entre ellas: 1. La utilización de L1 se puede convertir en un hábito en el que ambos (alumno - maestro) pueden acudir cuando una dificultad es encontrada. 2. L1 puede convertirse en un distractor cuando se aprende un lenguaje, aunque exista un sistema de lenguaje universal, el idioma difiere algunas veces. 3. Cuando se utiliza L1 en la enseñanza de lenguaje extranjero, errores pueden ocurrir en la transferencia del idioma. Ejemplo, en la transición del vocabulario a la gramática. Estudiantes cuya lengua materna es el español se les puede presentar la palabra "actualmente" y la palabra en inglés "actually". A pesar de la similitud el significado de ambas difiere. En español la palabra significa "ahora, en este preciso momento" mientras que en ingles la palabra significa "realmente". 4. El uso del L1 en las clases de EFL obstaculiza la recepción comprensiva de información un pre-requisito para el aprendizaje de cualquier idioma.

\section{El método bilingüe}

El método monolingüe ha sido criticado por muchos profesores de idiomas, los que encuentran la utilización del L1 en las clases de Inglés como Lengua Extranjera (EFL) beneficiosa en muchos niveles. Este punto de vista es expresado claramente por Deller y Rinvolucri (2003) y anteriormente por Atkinsong (1991). Más recientemente Widdowson (2003) llamado por su expresividad en el método bilingüe.

La lengua nativa por mucho tiempo ha sido considerada como un lenguaje bajo y lleno de errores. Este punto de vista es ahora criticado porque los profesores de Inglés como Lengua Extranjera (EFL) se han dado cuenta del significado del uso de la lengua materna dentro del aula de clase. Vivian Cook (2001) escribe de la lengua materna en las aulas EFL como: "a door that has been firmly shut in language teaching for over a hundred years" una puerta que ha sido cerrada en la enseñanza de idiomas por más de cien años.

Desde nuestra experiencia docente, hemos visto que los estudiantes cuando entran en el aula de clases no vienen inesperadamente en blanco, entran llenos de su lenguaje materno y herencia cultural la cual no se puede negar y ni menospreciar.

Los maestros de inglés como Lengua Extranjera que enseñan a estudiantes monolingües en el nivel más bajo de conocimientos del idioma Inglés, encuentran que la prohibición del uso de la lengua materna es prácticamente imposible. Por eso, en vez de mirar como una desventaja el uso de la lengua materna y pasado cultural de una forma inferior o una fuente de errores, deberían ser utilizados como una herramienta para maximizar el aprendizaje de una lengua extranjera.

Vale la pena señalar, que el uso de L1 dentro del aula de clases en una "rehabilitación" para esos estudiantes "que se ven forzados rápidamente a acudir y en algunos casos a esconder su diccionario bilingüe para no pasar la pena de ser señalados por usar su lengua materna”. La lengua 
materna representa un recurso poderoso que puede ser utilizada en un sinnúmero de formas para fomentar el aprendizaje, pero siempre debería ser utilizada de una manera correcta para maximizar el aprendizaje, en este caso del inglés.

\section{Uso consecuente de $\mathrm{L} 1$ en el aprendizaje de una lengua extranjera}

El uso de L1 no es el problema. El problema es Cuándo y Cómo utilizarlo. Antes de contestarnos estas preguntas se debe de tener en cuenta que el uso de L1 debe ser considerado como un medio para un fin. Auerbach (1993:19) concluye que "iniciando con el L1 provee un sentido de seguridad validando la experiencia del estudiante, permitiéndole expresarse lo que permite la aceptación a experimentar y tomar riesgos a la hora de hablar el Inglés”.

El lenguaje estudiado debe ser utilizado siempre que sea posible y el L1 cuando sea necesario. En los siguientes puntos hablaremos de algunos ejemplos apropiados en el uso de la lengua materna en las clases de inglés. Según Rhalmi (2009) pueden definirse como:

- Beginners (Principiantes): La lengua materna puede ser más que beneficiosa, cuando ellos progresen en su aprendizaje la lengua en estudio tomara la delantera.

- L1 puede reducir el tiempo: En lugar de pasar mucho tiempo en la explicación en Inglés, algunas veces es más fácil y efectivo dar la traducción del nuevo vocabulario o dar la explicación de los puntos gramaticales. Imaginémonos a un profesor que quiere introducir la palabra "truck" a estudiantes de la carrera de Ciencias Sociales iniciando la explicación de esta manera " a truck is a road vehicle with an engine, four wheels, and seats for a small number of pasangers" mientras que una simple traducción de la palabra (o talvez utilizando ayuda visual) sería más que suficiente.

- Comparación: Comparando el Inglés con la lengua materna puede ser una experiencia enriquecedora para los estudiantes. De hecho, descubrir las similitudes y diferencias de ambos idiomas puede mejorar la adquisición del idioma (Inglés).

Esta comparación se puede dar en diferentes niveles:

- Vocabulario

- Explorando los matices de la palabra en ambos idiomas.

- Construyendo mapas semánticos bilingües.

- Gramática

- Una comparación entre la gramática de L1 y la gramática en Ingles provee resultados interesantes.

- Esta comparación resalta la diferencia entre ambos idiomas. Maestros y estudiantes pueden construir estas diferencias para evitar una transferencia negativa (la transferencia de L1 puede resultar en errores)

- La comparación también muestra similitudes, las que sin duda alguna enriquecerán la internalización de la gramática del idioma estudiado. 
- Cultura: El idioma es un vehículo de los aspectos culturales. Si un maestro prohíbe el uso de L1, esto conlleva un concepto ideológico en la cultura L1 como inferior. Pero alternativamente, las diferencias culturales y similitudes pueden ser subrayadas para ayudar a los estudiantes a tolerar las diferencias, mientras que al mismo tiempo preservar su cultura única. Esto se puede lograr a través de varias actividades donde la lengua materna juegue un papel importante.

- Proverbios: Dando a los estudiantes una lista de proverbios en Inglés y preguntarle que encuentren su similar en L1 si es que existen. Si no, ellos trataran de encontrar la traducción en su lengua materna.

- Expresiones Idiomáticas: Otra vez, encontrar la expresión correspondiente o traducción de la palabra en Ingles, esto puede ser beneficioso para detectar las diferencias o similitudes en la cultura.

- Songs (canciones): Traducción de canciones.

- Chistes: En este punto la utilización de chistes en las actividades de EFL los estudiantes pueden traducir y contar o actuar los chistes en Ingles para crear un ambiente libre de estrés dando lugar a las especificaciones culturales.

- Estrés: La utilización de la lengua materna puede dar una sensación de seguridad y reconocer la identidad del estudiante, permitiéndole minimizar el estrés que pueden sentir dentro del aula de clases. Con el uso cuidadoso de la lengua materna los estudiantes pueden motivarse a experimentar y tomar riesgos en la utilización del inglés.

- Necesidades: Las necesidades de los estudiantes se pueden expresar en L1 debido a que todavía no manejan correctamente el inglés. Lo estudiantes no pueden expresar y comunicar sus necesidades con un idioma que manejan muy pobremente.

- Manejo del aula de clase: El manejo de la conducta y la disciplina se vuelve, algunas veces, difícil de manejar en inglés. Sin embargo, si un problema mayor emerge dentro del aula de clase, ¿Insistirá el maestro en la política de solo inglés para lograr controlar el acontecimiento?

- Gramática: L1 puede ser de gran ayuda cuando se enseña gramática. Por ejemplo, ejercicios de traducción pueden ser la práctica perfecta cuando hay un punto en gramática que está causando problema en los estudiantes.

- Instrucciones: De acuerdo a mi experiencia con las clases de EFL, me atrevo a decir que muchos errores en las evaluaciones se dan debido al poco entendimiento de las instrucciones a seguir para completar dicha tarea. La utilización de L1 puede re-direccionar esta inquietud, ayudando a los estudiantes a realizar lo que se les está solicitando.

- La Lógica: Los estudiantes deberán de entender la razón fundamental de las actividades o métodos. Es de suma importancia que ellos sepan donde iniciar y que es lo que podrán realizar. Esto solo se puede lograr en este nivel con el lenguaje nativo de los estudiantes.

\section{CONCLUSIONES}

El debate sobre la utilización de la lengua materna en el aula de clase todavía no se ha cerrado. Por un lado, están los maestros que rehúsan la utilización del lenguaje materno del todo y no reconocen ningún significado potencial en su utilización. 
Por otro lado, están estos maestros que abusan inmensamente de la sobreutilización de lenguaje materno. Ambos están abusando de un recurso de mucha importancia y se dedican cada uno por su propio camino.

Mi punto de vista como docente, y la experiencia que he adquirido en estos 3 años como docente de Inglés para esta Facultad me deja con la idea que consiste en la utilización del Inglés como un medio de instrucción siempre que sea posible y cambiar a lenguaje materno cuando realmente sea necesario. Cabe mencionar que un uso racional y juicioso del lenguaje materno dentro de las aulas de clase de Inglés como lengua extranjera puede ser más que ventajoso para lograr cumplir con los objetivos propuestos.

"If you talk to a man in a language he understands, that goes to his head. If you talk to him in his own language, that goes to his heart."

Nelson Mandela

\section{BIBLIOGRAFÍA}

Atkinson, D. 1991. The mother tongue in the classroom, a neglected resource? ELT Journal, 41, 4, pp. 241-47.

Auerbach, E. 1993. Reexaming English only in the ESL classroom. TESOL Quarterly 27, 1, pp. 9-32.

Cook, V (2001) Using the first Language on the classroom” Retrieved November 16, 2017, from http://www.viviancook.uk/SLA/Multicompetence/UsingL1.htm

Deller, S \& Rinvolucri, M (2002) “Using the Mother. Tongue: Making the Most of the Learner's Language". London: First Person Publishing.

Krashen, S. (1981) "Second Language Acquisition and Second Language Learning” University of Southern California, 5: 64-69

Own language use in ELT. (2016, November 22). Retrieved October 7, 2017, from https:// englishagenda.britishcouncil.org/continuing-professional-development/teacher-educatorframework/understanding-teaching-context/own-language-use-elt

Pellón, R. T., Miranda, D. R., \& Reyna, H. C. (2007). El uso de la lengua materna en la enseñanza de idiomas extranjeros. Retrieved November 16, 2017, from http://scielo.sld.cu/scielo. php?script=sci_arttext\&pid=S0864-21412007000300003

Using the native language in the English Classroom. (2014, August 25). Retrieved November 5, 2017, from http:/www.myenglishpages.com/blog/11-efl-classes/

Widdowson, H.G. (2003) „"Expert beyond experience”: Notes on the appropriate use of theory in practice. In. D. Newby (ed.) Mediating between Theory and Practice in the Context of Different Learning Cultures and Languages. Strasbourg/Graz: Council of Europe Press. 